\title{
Impact Loads on the Occupant under the Protection of an Inversion Tube Energy Absorber during a Helicopter Crash
}

\author{
Yunhua Luo ${ }^{1, a^{*}}$, Mengqi Wang ${ }^{1, b}$, Tao Yan ${ }^{2, c}$ and Lingjie Wang ${ }^{2, d}$ \\ ${ }^{1}$ School of Materials Science and Engineering, Huazhong University of Science and Technology, Wuhan 430074, \\ China \\ ${ }^{2}$ Avic Aerospace Life-Support Industries . LTD
}

\begin{abstract}
The objective of this paper is to investigate the impact loads on the occupants' head, neck, and spine under the protection of an inversion tube energy absorber during a helicopter crash landing. Due to the high vertical acceleration, the head, neck and spine are the most vulnerable parts of a body, so that an energy absorber is needed to dissipate the kinetic energy of the occupant and the seat to minimize the impact loads. In this paper, an inversion tube was adopted as an energy-absorbing device. The occupant injury conditions were evaluated by a numerical simulation. The result indicates that the impact loads on occupant's head, neck and spine are below the regulated thresholds under the protection of the energy absorber when the helicopter crash at a speed of $12.81 \mathrm{~m} / \mathrm{s}$ in vertical direction. As a consequence, the design of the occupant protection system has been proven reliable.
\end{abstract}

\section{Introduction}

Occupant protection is one of the most basic requirements for the helicopter seat structure design. During a crash landing, the impact loads may cause fatal injury on occupants' head, neck and spine [1]. To attenuate the impact loads on the occupant, the seat must process enough energy-absorbing capacity to dissipate the kinetic energy of the occupant and the seat before it bottoms out. Amount of work has been done by many researchers to develop various methods to meet the requirements of occupant safety. Michle Guida et al [2] proposed an innovative method of integrating anticrash energy absorbers made of a new composite material into landing gears to absorb the kinetic energy of the seat and the subfloor of a helicopter. S.A. Galehdari et al [1] developed a graded honeycomb shock absorber to attenuate the impact loads on the occupant to an acceptable level. Zhang [3] designed a sliding seat for preventing the neck from severe injury. However, there is no better way of attenuating the impact loads on the occupant than using energy-absorbing devices. The inversion tube is one of the most commonly used energy absorber for its excellent performance in absorbing energy, high manufacturability and low cost [4].

Considerable investigations have been conducted on the physical properties of the inversion tube energy absorber, however, little information exists on the impact loads on the occupant with an inversion tube installed on the seat. This study investigated the injury conditions of occupants' head, neck and

\footnotetext{
${ }^{\mathrm{a}}$ Corresponding author : yhluo@hust.edu.cn, ${ }^{\mathrm{b}} 1406472367 @ q q . c o m,{ }^{c} 2453926883 @ q q . c o m$,

d353229291@qq.com
} 
spine during a helicopter crash by a numerical simulation conducted by MSC.Dytran, an explicit code, associated with the articulated total body (ATB) program.

MSC.Dytran is good at solving nonlinear transient impact problems, while the ATB program is commonly used for computing the gross body response in various dynamic environments. The process of this simulation is not a procedure for running one program, then transferring information and running the other, these programs run concurrently and exchange data as the other proceeds. The ATB model used in this simulation consists of 17 rigid segments connected by 16 joints and takes on a tree-like structure [5]. The mass and inertia moment of each segment are equivalent to human counterparts, and the properties of the dummy model joints are similar to the corresponding human articulations. Besides, some shell elements with negligible density are covered on the dummy to provide a correct body contour for a contact calculation.

In this study, much endeavor was made to obtain the impact loads on the occupant and evaluate occupant injury conditions. The following proceeds as follows: the inversion tube used in this occupant protection system is introduced in section 2, section 3 shows how the whole model of the seat/occupant system was established, the results were showed and discussed to evaluate the occupant injury conditions in section 4, in the final section, conclusions were drawn and prospects were made for future research.

\section{Inversion tube energy absorber.}

The inversion tube is one of the most significant parts of the shock-mitigation system. The kinetic energy of an occupant/seat system can be dissipated rather than stored by the plastic deformation of an inversion tube. Figure 1 shows the process of the inversion tube deformation under the axial compressive force. It is obvious that the deformation part constantly keeps circular in the process of deformation from A to A'. Therefore, the deformation force keeps fixed, which provides the occupant with a steady deceleration process.

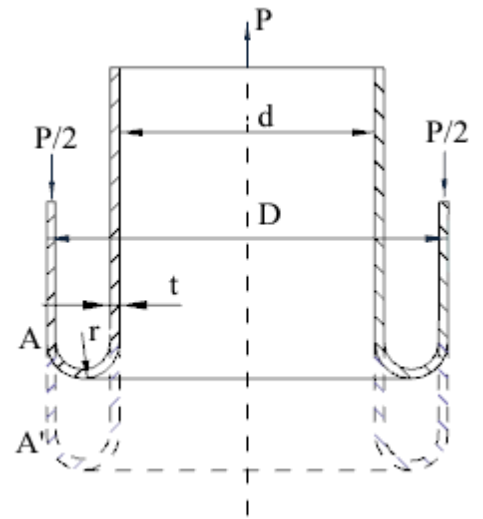

Figure 1. The deformation process of an inversion tube

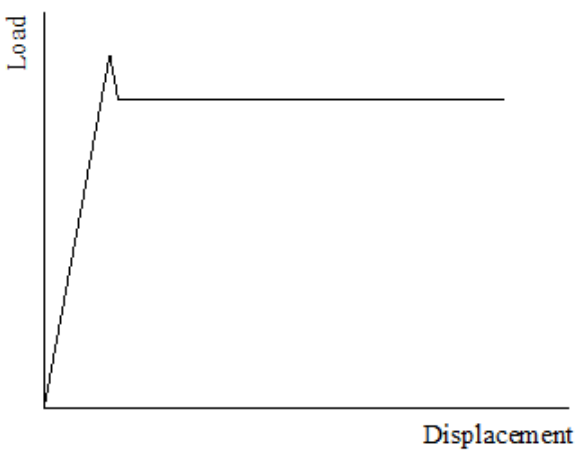

Figure2. Load-displacement curve of an inversion tube

Figure 2 shows the Load-displacement curve of an inversion tube. The load rises rapidly to its peak value, after that the deformation force descends a little and then keeps fixed until the inversion tube strokes out. The deformation force of the inversion tube can be depicted as the following equation:

$$
\mathrm{P}=\frac{\pi D \sigma_{s} t}{\sqrt{3}}\left[\ln \frac{D}{d}+\ln \left(1+\frac{t}{2 r}\right)\right]
$$

Where $t, r, d$ and $D$ respectively represent the thickness, knuckle radius, inside diameter and outside diameter of an inversion tube, showed as Figure 1. And $\sigma \mathrm{s}$ is the yield stress. In this research, $1 \mathrm{Cr} 18 \mathrm{Ni}$ TTi was selected as the material of the inversion tube and its physical properties are showed in Table 1. 1Cr18Ni9Ti is known for its high plasticity, tenacity, and weldability. 
Table 1.The properties of $1 \mathrm{Cr} 18 \mathrm{Ni} 9 \mathrm{Ti}$

\begin{tabular}{|c|c|c|c|}
\hline Name & Density $\left(\mathrm{kg} / \mathrm{m}^{3}\right)$ & Elastic modules (GPa) & Position ratio Yield stress (MPa) \\
\hline $1 \mathrm{Cr} 18 \mathrm{Ni} 9 \mathrm{Ti}$ & 7850 & 202 & 330 \\
\hline
\end{tabular}

\section{Finite element model}

The numerical model of the seat/occupant system showed in Figure 3 was established in MSC.Patran, a pre-processing software. The redundant seat structures that exert little influence on simulation results were taken out to improve the computational efficiency. The seat consists of 5 major parts: an energy absorber, two guides, a seat bucket, belts and a pedal. The inversion energy absorber is used to prevent the occupant from severe injury. The guides transform occupants' motion to vertical movement relative to the subfloor. The simplified seat bucket is used to define a contact between the seat and the dummy. The belt is designed as a 4-point belt to offer the occupant a symmetric constraint. The pedal constraints the foot and legs from a large movement. Excluding the dummy, the complete model includes 9788 nodes, 4200 HEXA elements, 1024 QUAD elements and 142 beam elements.

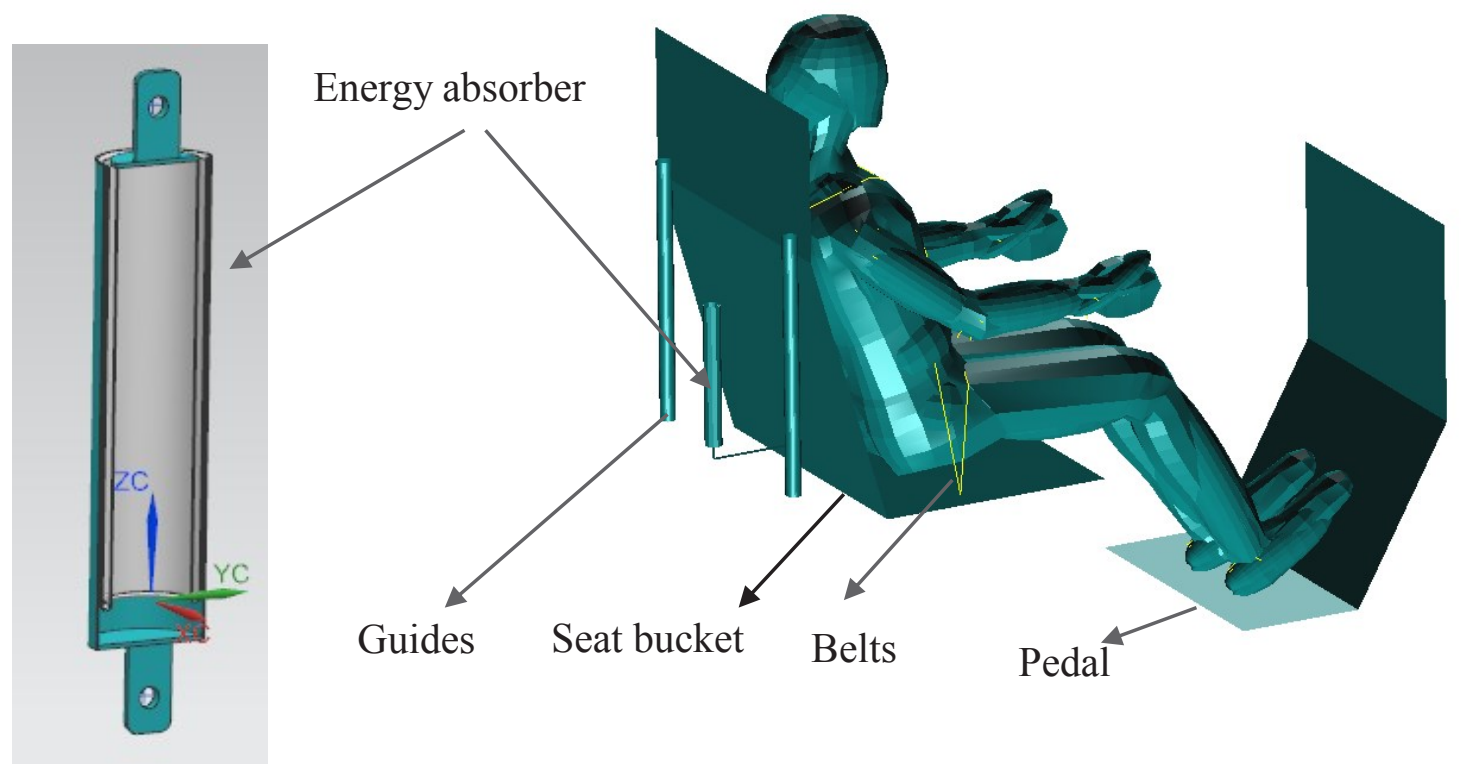

Figur3. Finite element model

A FAA 50th-percentile Hybrid-III dummy was selected in this model to maximize the effectiveness over the range of occupant weight. The hybrid-III dummy has a large improvement over the hybrid-II dummy on the characteristics of the neck, chest, and knee and became the only test dummy regulated by the FMVSS208 (Federal Motor Vehicle Safety Standards). The dummy was seated in normal upright position and located in the middle of the seat to keep a symmetric model.

The initial velocity of the whole model was set at $12.81 \mathrm{~m} / \mathrm{s}$ for a statistical fact that the initial vertical velocity is smaller than $12.81 \mathrm{~m} / \mathrm{s}$ among $95 \%$ helicopter crash accidents. The seat input deceleration pulse is a triangle shape with a peak value of $30 \mathrm{G}$ and a span time of $0.087 \mathrm{~s}$. The boundary conditions are not complicated, however, two points must be noted in the process of preparing the simulation: 
One is the contact between the bushes and the pillars. Both of the guides and the pillars are rigid bodies, so that the pillars are easy to penetrate the bushes, which may cause the inversion tube to deform unstably. Two methods are suggested to prevent this penetration:

(1) Set a small penetration check distance.

(2) Define a large contact force scale factor.

The other needed to be noted is the time step. Too small time step may result in too long computational time or even quitting from the simulation. The critical time step can be calculated by the following equation:

$$
\nabla t_{c r i}=\frac{S L}{C}
$$

Where $\mathrm{S}$ is the time step factor, whose default value is $0.66, \mathrm{C}$ is the sound speed of this material, and $\mathrm{L}$ is the smallest dimension in this model. Therefore, a small dimension in the model will generate a small time step and result in solving difficulty or even bringing the simulation to a halt. In the model of this simulation, the smallest dimension is the thickness of the inversion tube which must be controlled at an acceptable level.

\section{Results and discussion}

\subsection{Occupant responses}

The process of occupant response in the simulation is presented in figure 4 . The result indicates that there is no large movement on the body except hands, which may be attributed to the belt constraint. The hands move largely so that they are easy to collide with the helicopter instruments and get fractured. Besides, it is found that the inversion tube deformed stably until it bottoms out.
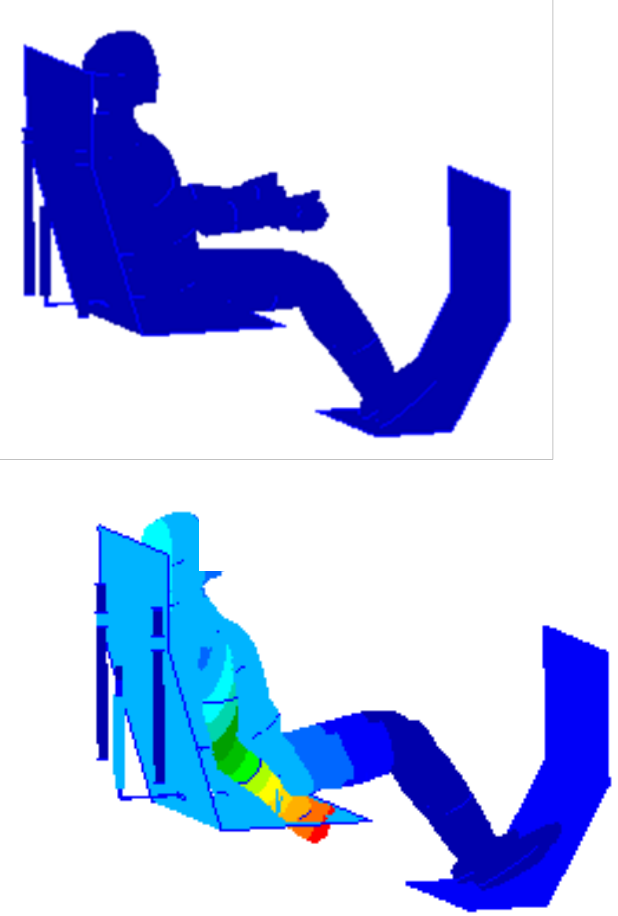
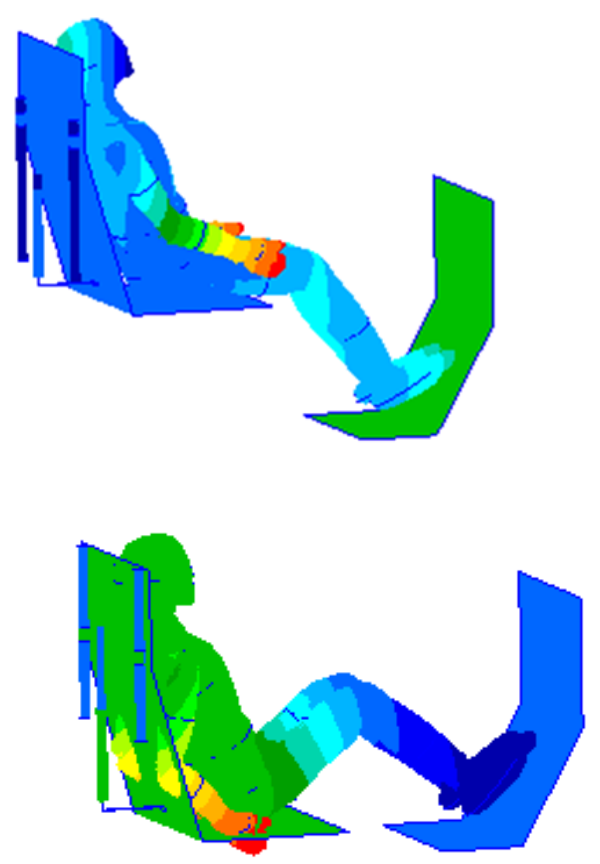

Figure 4. Occupant responses in the environment of $12.81 \mathrm{~m} / \mathrm{s}$ initial velocity and $30 \mathrm{G}$ deceleration pulse 


\subsection{Head injure criteria}

The National Highway Traffic Safety Administration (NHTSA) has established several head injure evaluation criterions, foremost among them is the Head Injure Criteria (HIC) that has been accepted by FMVSS208 as a head impact tolerance specification. It regulates that the HIC value should be less than 1000 , or the head will suffer a severe injury. The HIC value can be calculated by the following equation:

$$
\mathrm{HIC}=\max \left[\left(t_{2}-t_{1}\right)\left\{\frac{1}{t_{2}-t_{1}}\left(\int_{t_{1}}^{t_{2}} a(t) d t\right)^{2.5}\right\}\right]
$$

Where $t_{1}$ and $t_{2}$ respectively represent the starting and ending time, during which time the HIC value reaches to its maximum value. Figure 5 shows the HIC value of the occupant during the crash. The maximum HIC value is 325 , occurring at $35 \mathrm{~ms}$ and under the HIC threshold. As a result, the occupant head is safe under the protection of the alleviation system.

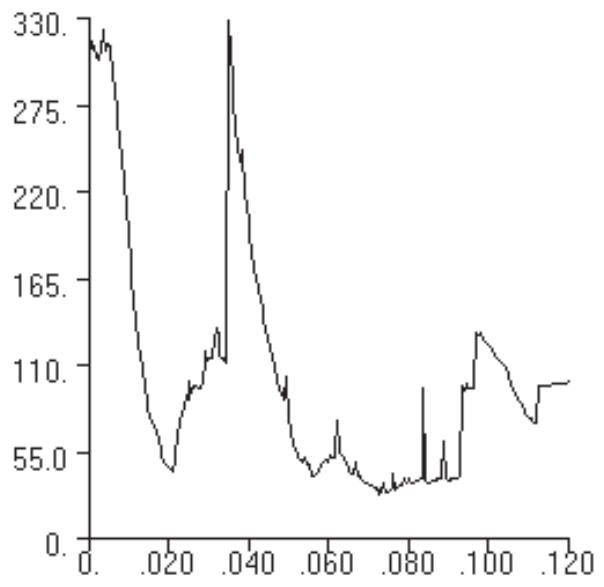

Figure 5. The HIC curve

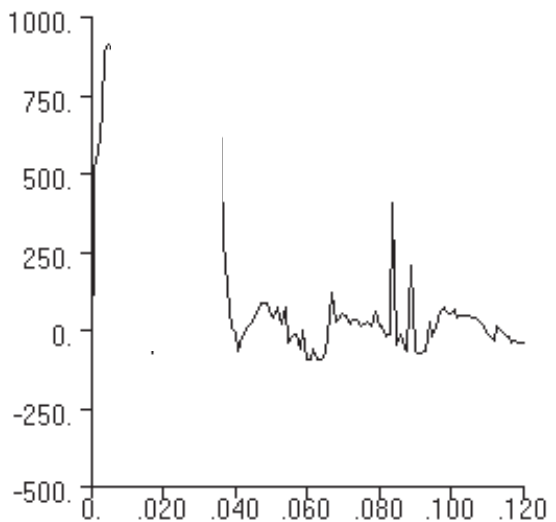

Figure 6. The neck load of the occupant

\subsection{Neck load}

The neck is a vulnerable part of a body and the impact load on the neck should be under $1100 \mathrm{~N}$ or the neck may get injured. From figure 6, it can be found that the maximum neck load is $909 \mathrm{~N}$ at $0.007 \mathrm{~s}$, below the neck load threshold. Therefore, the neck will not be objected to a severe injury.

\subsection{Spine load}

The spine is the main segment that supports the body weight. It consists of many movable sclerotomes and the motion of a spine is a result of the associated movement of all sclerotomes. The spine will bent when it is objected to a large compressive force.

Chandcer and Shanahan developed a lumbar load criterion that the maximum spine load should be no more than $6675 \mathrm{~N}$, which is now integrated into the Federal Aviation Regulation (FAR) [6].

A spine consists of many segments, among which the lumbar spine bears the maximum load, $50 \% \sim 60 \%$ body weight. At the circumstance of $30 \mathrm{G}$ impact acceleration pulse in vertical direction and without any energy-absorbing device, the maximum lumbar spine load can be roughly calculated by the following equation:

$$
\mathrm{F}=0.6 \mathrm{~m} 50^{\text {th }} \mathrm{a}
$$


Where $\mathrm{m} 50^{\text {th }}$ is the mass of a $50^{\text {th }}$-percentile occupant. The mass of a $50^{\text {th }}$-percentile hybrid III dummy is $76.23 \mathrm{~kg}$. From Eq.4, we can obtain that the lumbar force is $13447 \mathrm{~N}$ under the impact acceleration of $30 \mathrm{G}$, which is far more than the spine load-carrying capacity. As a consequence, an inversion tube energy absorber is needed to attenuate the lumbar force to an acceptable level.

Figure 7 shows the lumbar compressive force of the dummy. The result indicates that the lumbar compressive force rises rapidly to its peak value, $4855 \mathrm{~N}$, at $0.006 \mathrm{~s}$, and then decreases sharply to $181 \mathrm{~N}$ at $0.017 \mathrm{~s}$, after that it keeps no more than $3000 \mathrm{~N}$ until the end. It can be concluded that the spine will not get injured for the inversion tube alleviates the impact load on occupant spine.

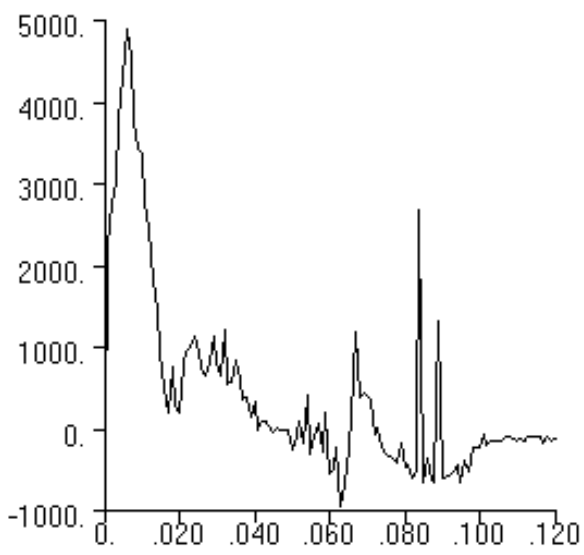

Figure 7. The lumbar compressive spine load

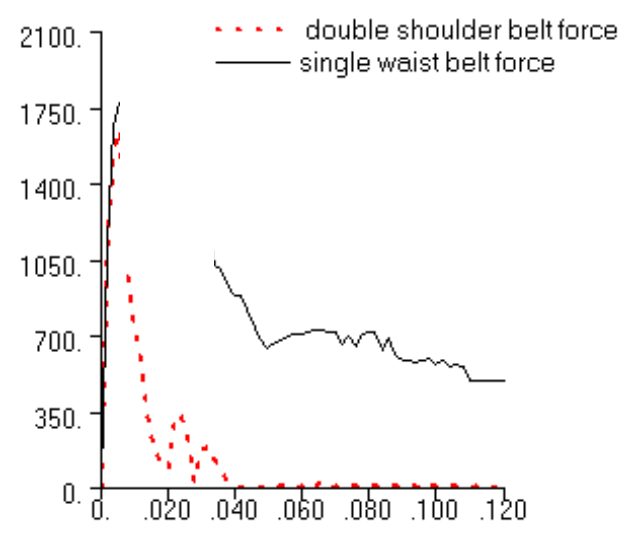

Figure 8. Seat belt load

\subsection{Seat belt load}

The seat belt consists of two shoulder belts and a waist belt. The CCAR23.562 regulates that the double shoulder belt load should be no more than $8900 \mathrm{~N}$ while the single waist belt load should be less than $7790 \mathrm{~N}$. The shoulder belt load and waist belt load are showed in Figure8. The maximum shoulder belt load is $1645 \mathrm{~N}$ and the maximum waist belt load is $2024 \mathrm{~N}$. The load on both of the two kinds of belts meets the requirements of CCAR23.562. Besides, it is found that both the two kinds of belts play a significant role on preventing the dummy from strenuous movement from the beginning to $40 \mathrm{~ms}$, after that only the waist belt works and takes effect.

\section{Conclusions}

To evaluate the reliability of the impact-alleviation system design, the occupant injure severity was investigated by a numerical simulation. It can be concluded that the inversion tube design is reliable, for that the HIC value, impact loads on the neck and the spine and belt force are all under the regulated thresholds under the protection of the shock-mitigation system.

This study is also committed to providing a reference for the inversion tube energy absorber design. The inversion tube parameters, such as the knuckle radius, outside diameter, inside diameter, thickness, length, material type and so on, can be changed and analyze the impact loads on occupant under the protection of different inversion tubes to optimize the energy-absorbing energy design. Besides, two things must be paid attention to:

(1) Owing to the fact that other seat structures, such as the cushion, take effect on attenuating the impact loads on the occupant but they were neglected in this simulation, the impact loads on occupant in this simulation results are a little larger than the loads in the real environment. 
(2) This simulation only takes the velocity and the acceleration in the vertical direction into account. But the real helicopter crash environment is much more complicated. The model processes the velocity and the acceleration in the lateral and forward direction during a helicopter crash.

This research is just a preliminary investigation on the occupant protection system during a helicopter crash. To further this study, more factors, such as the cushion, initial velocity and acceleration in the forward and lateral direction should be considered.

\section{Acknowledgements}

The work described in this paper is financially supported by the Avic Aerospace Life Support Industries .LTD. under grant number 20142979004. The authors wish to gratefully thank this support.

\section{References}

1. S.A. Galehdari and H. Khodarahmi, Design and analysis of a graded honeycomb shock absorber for a helicopter seat during a crash condition, International Journal of Crashworthiness, 2016 21(3), 231-241.

2. Michele Guida, Francesco Marulo, Bruno Montesarchio and Massimiliano Bruno, Innovative Anti Crash Absorber for a Crashworthy Landing Gear, Appl Compos Mater , 21(2014) pp:483-494.

3. Xiaowei Zhang and Qing Zhou, An energy-absorbing sliding seat for reducing neck injury risks in rear impact — analysis for prototype built, Traffic Injury Prevention, 2016, 17(3), 313-319.

4. Kai Yang, ShanqingXu, JianhuShen, ShiweiZhou and YiMinXie, Energy absorption of thin-walled tubes with pre-folded origami patterns: Numerical simulation and experimental verification, Thin-Walled structures, 103 (2016), pp:33-44.

5. Huaining Cheng,Annette L. Rizer, Articulated Total Body Model Version V User's Manual, Biodynamics and Protection Division Human Effectiveness Directorate Air Force Research Laboratory,1998. Engineering, 2(4), 2012, 562-577.

6. Rasoul Moradi, Hamid K. Beheshti and Hamid M. Lankarani, Lumbar load attenuation for rotorcraft occupants using a design methodology for the seat impact energy-absorbing system , Central European Journal of Engineering, 2 (4) , 2012, 562-577. 